\title{
Pseudomonas arthritis treated with parenteral and intra-articular ceftazidime
}

\author{
KRYSTYNA WALTON, ${ }^{1}$ R C HILTON, ${ }^{1}$ AND RACHEL A SEN ${ }^{2}$ \\ From the ${ }^{1}$ Department of Rheumatology, University of Manchester, Hope Hospital, Salford M6 8HD, and \\ the ${ }^{2}$ Department of Microbiology, Hope Hospital, Salford M6 $8 H D$
}

SUMmARY A 73-year-old diabetic presented with septic arthritis of the knee; Pseudomonas aeruginosa was isolated. She was successfully treated with a combination of parenteral and intraarticular ceftazidime, after failure to eradicate the organism with adequate serum levels of gentamicin and full doses of azlocillin.

Key words: septic arthritis, crystals, intra-articular antibiotics.

Joint infections with Gram-negative bacilli are particularly difficult to treat and are associated with a poor eventual outcome. ${ }^{1}$ If synovial fluid culture remains positive for more than four days after initiation of appropriate antibiotic therapy, prognosis is also poor. ${ }^{2}$

\section{Case report}

A 73-year-old lady with late onset insulin dependent diabetes mellitus and peripheral vascular disease presented in February 1983 with a three-week history of pain and swelling of the right knee. Four years earlier a left sided below knee amputation was performed for vascular insufficiency. On examination the right knee was swollen, hot, and tender. 40 $\mathrm{ml}$ of turbid brownish fluid was aspirated. Microscopy showed 61380 white blood cells per $\mathrm{mm}^{3}$ $\left(61 \cdot 38 \times 10^{9} / 1\right)(96 \%$ polymorphs), numerous intracellular pyrophosphate crystals, but no organisms. On culture $P$. aeruginosa was isolated. Blood cultures were negative, and the organism was not isolated from any other site (including a large ischaemic ulcer just above the right lateral malleolus). $X$-rays showed chondrocalcinosis in the right knee.

Accepted for publication 6 February 1985.

Correspondence to $\mathrm{Dr} \mathrm{K}$ Walton.
The patient was treated with intravenous gentamicin and azlocillin $2 \mathrm{~g}$ every eight hours, to which the organism was sensitive. Gentamicin was given for two weeks but had to be stopped because of persistently high serum levels secondary to poor renal function. Azlocillin was continued for a total of five weeks, but despite adequate serum bactericidal activity pseudomonas was still being isolated from the joint fluid, and the knee remained hot and swollen. $X$-ray films at this time showed evidence of bone involvement, with erosion of the medial margin of the right upper tibia. Azlocillin was discontinued, and treatment commenced with ceftazidime, to which the organism was also sensitive, 1.5 $\mathrm{g}$ intravenously daily and $100 \mathrm{mg}$ intra-articularly, initially weekly increasing to three times per week. Within two weeks of starting this regimen there was considerable improvement in both the patient's clinical state and that of the infected knee. We continued this treatment for two months, and followed it with one month of intramuscular ceftazidime. At the end of this period the knee showed no signs of continuing infection and was pain free with a good range of movement. Serial joint aspirations were consistent with response to treatment, with a marked fall in the white cell count. There was no evidence of a recurrence of infection when the patient was reviewed six months later. 


\section{Discussion}

Septic arthritis due to Gram-negative organisms is still relatively uncommon, but there is evidence that the frequency is increasing. ${ }^{1}$ Intravenous drug use and impaired host defence are the two most important predisposing factors. ${ }^{34}$

Ceftazidime is a third generation cephalosporin, active against pseudomonas species and was then under trial. In this particular patient it was used because intravenous therapy with bactericidal serum levels of gentamicin and azlocillin had failed to eradicate the pseudomonas infection after five weeks, and this situation usually indicates a very poor prognosis. ${ }^{2}$

Experience of treating coexisting septic arthritis and crystal induced arthritis is very limited, as this association has been reported on only 13 occasions, five of which were due to Gram-negative bacilli. . $^{5-14}$

Intra-articular antibiotics are rarely given in the treatment of septic arthritis, as the penetration of parenteral and oral antibiotics across the synovial membrane into the synovial fluid is said to be good. ${ }^{15-17}$ We believe that this is the first occasion that ceftazidime has been administered intraarticularly, and though we have no evidence that it contributed to the eventual successful outcome, we feel this mode of administration should be seriously considered to supplement parenteral or oral therapy when factors such as coexistent pyrophosphate arthritis are likely to make eradication of infection difficult.

This case also illustrates the importance of synovial fluid culture even when crystals have been shown in an acutely inflamed joint.

We would like to thank Glaxo Laboratories Ltd for allowing us to use and supplying us with ceftazidime.

\section{References}

1 Goldenberg D L, Cohen A S. Acute infectious arthritis. A review of patients with non-gonococcal infections (with $\mathrm{em}-\vec{F}$ phasis on therapy and prognosis). Am J Med 1976; 60: 369-77.

2 Ho G, Su E Y. Therapy for septic arthritis. JAMA 1982; 247:O 797-800.

3 Goldenberg D L, Cohen A S. Arthritis due to gram-negative bacilli. Clin Rheum Dis 1978; 4: 197-210.

4 Gifford D B, Patzakis M, Ivler D, Swezey R L. Septic arthritis $\stackrel{\mathbb{Q}}{\Omega}$ due to pseudomonas in heroin addicts. J Bone Joint Surg [Am] ڤ్ 1975; 57: 631-5.

5 Hess R, Martin J. Pyarthrosis complicating gout. JAMA 1971; $\overrightarrow{0}$ 218: $592-3$.

6 Smith J E, Phelps P. Septic arthritis, gout, pseudogout and osteoarthritis in the knee of a patient with multiple myeloma. Oे Arthritis Rheum 1972; 15; 89-96.

7 Goldenberg D, Brandt K D, Cathcart E S, et al. Acute arthritis caused by gram negative bacilli: a clinical characterisation. Medicine (Baltimore) 1974; 53: 197-208.

8 McConville J, Pototsky R S, Calia F M et al. Septic and crystalline joint disease: a simultaneous occurrence. JAMA 1975; 231: 841-2.

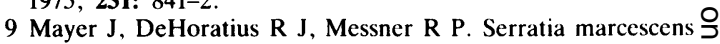
caused arthritis with negative and positive birefringent crystals. Arch Intern Med 1976; 136: 1323-5.

10 Jarrett M P, Grayzel A I. Simultaneous gout, pseudogout and septic arthritis. Arthritis Rheum 1980; 23: 128-9.

11 Edwards G S, Russell I J. Pneumococcal arthritis complicating $\overrightarrow{0}$ gout. J Rheumatol 1980; 7: 907-10.

12 Hamilton $M$ E. Parris J M, Gibson R S, Davis J S Simultaneous gout and pyarthrosis. Arch Intern Med 1980; 140: 917-9.

13 Heinicke M, Gomez-Reino J J, Gorevic P D. Crystal arthropathy as a complication of septic arthritis [letter]. J Rheumatol 1981; 8: 529-31.

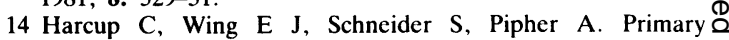
meningococcal arthritis and pseudogout in an elderly woman. $\overline{\vec{O}}$ Arthritis Rheum 1983; 26: 1409-11.

15 Nelson J D. Antibiotic concentrations in septic joint effusions. N Engl J Med 1971; 284: 349.

16 Chou A, Hecht R, Winters R. Gentamicin and carbenicillin penetration into the septic joint. $N$ Engl J Med 1971; 285: 178.

17 Sattar M A, Barrett S P, Cawley M I D. Concentration of some antibiotics in synovial fluid after oral administration with special reference to anti-staphylococcal activity. Ann Rheum Dis 1983; 42: $67-74$.

\section{Clinical vignette}

\section{Small muscle wasting?}

Is it small muscle wasting when you see hollows between the tendons on the back of the hands in rheumatoid arthritis? Depress the tendons and you will probably find that it is not. The extensor tendons often show the $\stackrel{\mathcal{S}}{\rightarrow}$ sign of 'tendon bridging', since they bridge from the swollen wrist to the swollen metacarpophalangeal joint.

(Readers are invited to submit brief accounts of new or little known physical signs in rheumatic diseases - Editor.) 